\title{
Transcatheter left atrial appendage closure in patients with atrial fibrillation
}

\author{
Comparison between non-dedicated and dedicated Amplatzer Devices \\ Michael Schmid ${ }^{a}$, Steffen Gloekler ${ }^{a}$, Ardan M. Saguner ${ }^{c}$, Andreas Wahl ${ }^{a}$, Urs Fischer ${ }^{b}$, Marcel Arnold $^{b}$, \\ Ahmed A. Khattab ${ }^{a}$, Fabian Nietlispach ${ }^{a}$,Enio Guerios ${ }^{a}$, Peter M. Wenaweser ${ }^{a}$, Stephan Windecker ${ }^{a}$, Bernhard Meier ${ }^{a}$ \\ a Cardiology, Bern University Hospital, Bern, Switzerland \\ b Neurology, Bern University Hospital, Bern, Switzerland \\ c Cardiology, Zurich University Hospital, Zurich, Switzerland
}

\section{Summary}

Background: Atrial fibrillation (AF) carries an increased risk of systemic thrombo-embolism. More than $90 \%$ of emboli in non-valvular AF originate from the left atrial appendage (LAA). Percutaneous closure of the LAA offers an alternative to long-term oral anticoagulation (OAC). Amplatzer devices have been proposed for this. A dedicated device (Amplatzer Cardiac Plug [ACP]) has been introduced to improve safety and efficacy compared to non-dedicated devices (NDAs, atrial and ventricular septal occluders) used previously for LAA closure.

Objective: The present study investigated procedural and clinical outcomes of LAA closure with the new ACP compared to NDAs.

Methods: All patients with LAA closure using an Amplatzer device at the Bern University Hospital, were entered into a prospective registry. Trans-oesophageal echocardiography (TEE) preceded LAA closure which was performed under local anaesthesia and fluoroscopic guidance only. Correct device position was documented by fluoroscopy and before discharge by trans-thoracic echocardiography (TTE). Follow-up TEE and clinical assessment by a neurologist were performed 3-9 months later. A later follow-up was performed by telephone contact.

Results: A total of 64 consecutive patients (NDA group n $=32$, June 2002 to December 2008; ACP group $\mathrm{n}=32$, January 2009 to March

Authors' contribution: The first two authors contributed equally to this work.

Funding / potential competing interests: $\mathrm{BM}$ received research grants and speaker fees from St. Jude-AGA. AK received proctor fees from St. Jude-AGA 2010) were included. The thromboembolic $\mathrm{CHADS}_{2}$ score risk was lower in the NDA group compared to the ACP group (1.0 \pm 1.0 versus $2.5 \pm 1.3, p=0.44)$. Despite a lower rate of combined interventions, total fluoroscopy time and total amount of contrast medium were higher in the NDA group ( $28 \pm 13 \mathrm{~min}$ versus $19 \pm 20 \mathrm{~min}, p=0.06$, and $424 \pm 182 \mathrm{ml}$ versus $226 \pm 134 \mathrm{ml}$, $p<0.01$, respectively). In the NDA group, the technical success rate was $84 \%$, compared to $100 \%$ in the ACP group $(p=0.02)$. Device embolisation occurred in $5(16 \%)$ patients in the NDA group but did not occur in the ACP group $(p=0.02)$. The incidence or suspicion of thrombus on devices were: $2 / 17(12 \%)$ in the NDA and $6 / 28(21 \%)$ in the ACP group $(p=0.10)$. During followup (mean $7.2 \pm 2.7$ months, NDA long-term follow-up $75 \pm 16$ months), no cerebrovascular accidents (CVA) occurred in either group.

Conclusion: The ACP facilitated the transcatheter LAA closure procedure and improved its safety compared to NDAs. It promises to enhance their favourable long-term results.

Key words: atrial fibrillation; stroke prevention; left atrial appendage; left atrial appendage closure

\section{Introduction}

Atrial fibrillation (AF) is the most common arrhythmia [1-3]. In Europe, the overall prevalence of AF amounts to $5.5 \%$ [1], increasing steeply with age $(>17 \%$ in patients over 85 years). The risk of stroke due to $\mathrm{AF}$ increases with the $\mathrm{CHADS}_{2}$ score [4]. The more elaborate $\mathrm{CHA}_{2} \mathrm{DS}_{2}$-VASc score features one additional point each for vascular disease, age between $65-74$ years and female gender, and two points for age $>75$ years [5]. More than $90 \%$ of emboli in non-valvular AF originate from the left atrial appendage (LAA) [6-9]. Optimal prophylaxis of systemic thrombo-embolism in patients with AF is warranted and significantly improves prognosis [10-13].

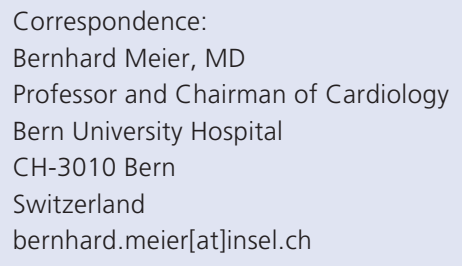


Oral anti-coagulation (OAC) currently using vitamin $\mathrm{K}$ antagonists represents the gold standard to prevent cerebrovascular accidents (CVAs) and systemic thrombo-embolism in patients with $\mathrm{AF}$ or atrial flutter $[10,11]$. However, OAC carries a yearly risk of relevant bleedings, ranging from 0.9-2.7\% [14]. Furthermore, the target international normalised ratio (INR) value of 2.0-3.0 proved maintained in $<50 \%$ of patients [15]. Acetylsalicylic acid (ASA), even when combined with

\section{Figure 1}

Left atrial appendage (LAA) occlusion with Amplatzer Occluder designed for septal occlusion (A) and the dedicated Amplatzer Cardiac Plug (B).

$\mathrm{LA}=$ left atrium.

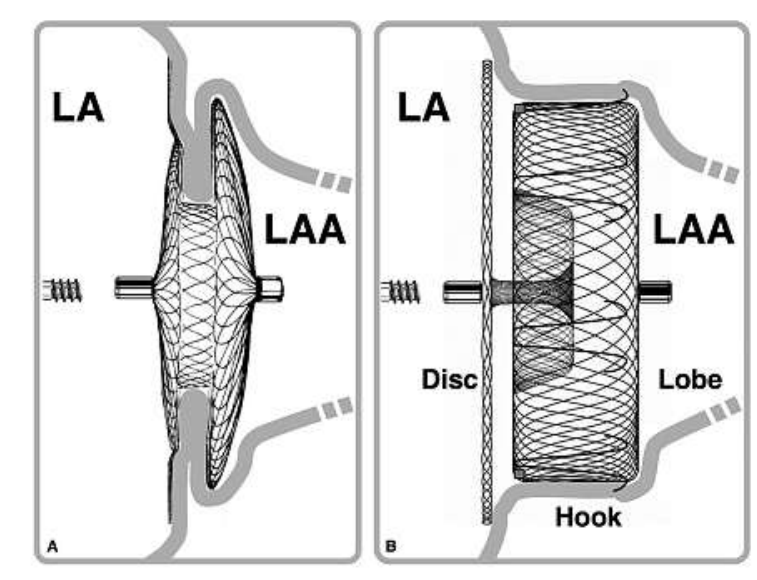

clopidogrel, is less effective in stroke prevention than OAC [16] and therefore, (dual) anti-platelet therapy is not recommended as an alternative to $\mathrm{OAC}$ in patients with a $\mathrm{CHADS}_{2}$ score $>1$.

Since 1948, exclusion of the LAA has been performed during cardiac surgery, particularly in mitral valve surgery with a high prevalence of $\mathrm{AF}[7,17,18]$. Trans-catheter closure of the LAA was first introduced in 2001 [19]. The device used then (PLAATO) was abandoned in 2006. An alternative method was established in June 2002 by use of a variety of non-dedicated Amplatzer occluders (NDAs, St. Jude, Plymouth, MS, USA) [20] and has been modified with a dedicated device (Amplatzer Cardiac Plug [ACP]) in 2008 [21] (fig. 1-3).

In 2009, a first prospective randomised trial (PROTECT AF) compared a third technique using the WATCHMAN device (Boston Scientific, Plymouth, MN, USA) with OAC and proved non-inferiority of trans-catheter LAA closure [22].

In the present study, NDAs (atrial septal defect (ASD), ventricular septal defect (VSD), patent foramen ovale (PFO) Amplatzer occluders) and the new ACP were compared with regard to procedural and followup data. The initial rationale for the use of ASD, PFO and VSD devices was derived from their established user-friendliness, low thrombogenicity, high potential for adaptability to the widely varying anatomy of the LAA, and also the desire to offer an alternative to patients without protection due to lack of anticoagulation beyond the complex PLAATO procedure. The occa-

Figure 2

Trans-oesophageal echocardiography of the left atrial appendage (LAA) before (A) and after (B, C) occlusion with an Amplatzer Cardiac Plug.

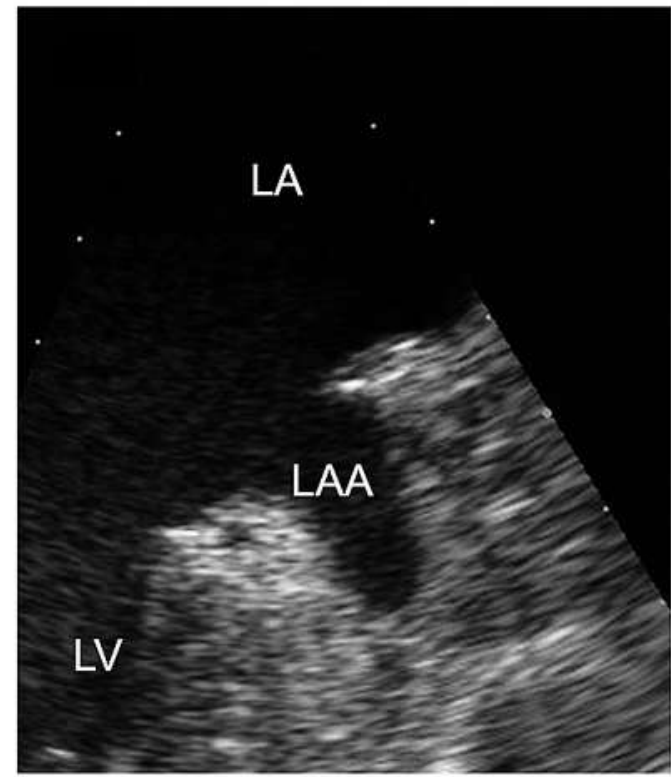

A

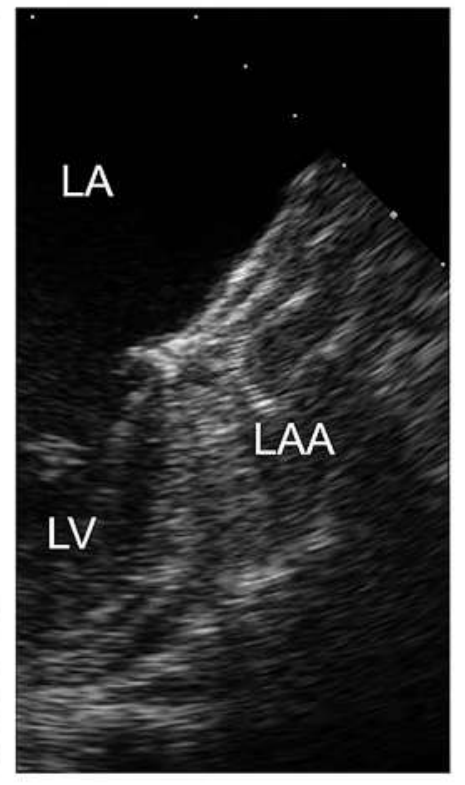

B

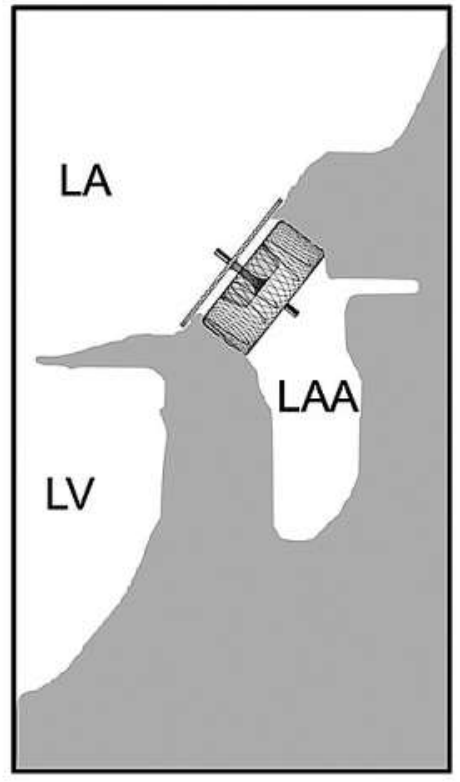

C 


\section{Figure 3}

Left atrial appendage (LAA) occlusion according to the pacifier principle ( $A, C$, the disc sits outside the LAA like the pacifier plate outside a toddler's mouth, B) using a non-dedicated Amplatzer Occluder (20 mm ASD occluder) depicted in a cadaver heart (D) with a $32 \mathrm{~mm}$ visible disc.

$\mathrm{LA}=$ left atrium.

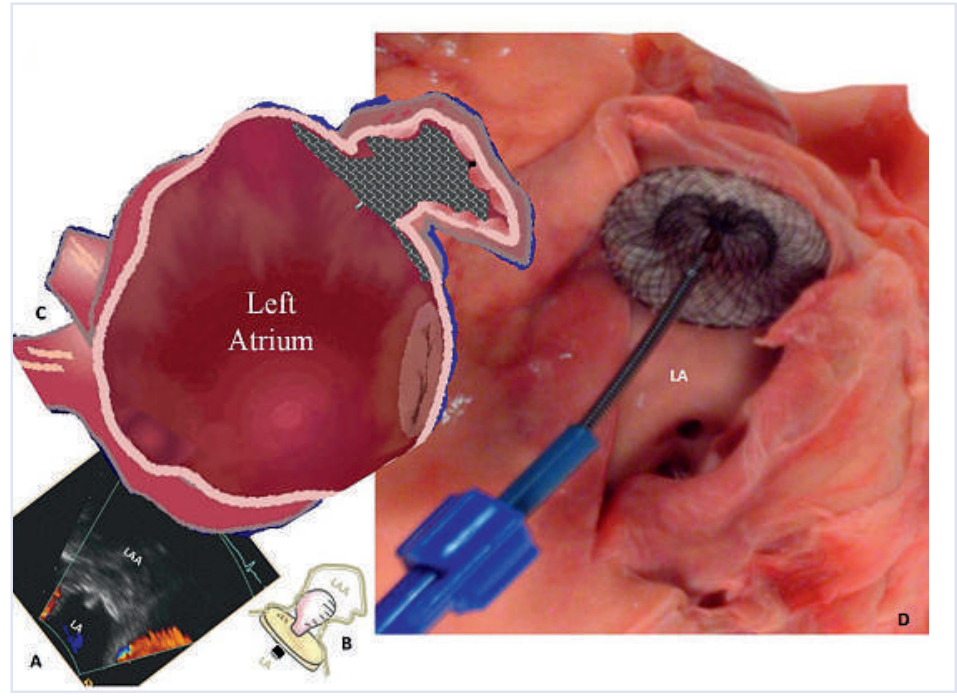

sional experience of having to retract such devices from the LAA when inadvertently deployed too far away from the inter-atrial septum while attempting closure of wall defects, instigated the positioning of them there on purpose for LAA occlusion.

\section{Methods}

\section{Study design}

All patients who received an Amplatzer transcatheter LAA occlusion device since 2002 at the Bern University Hospital, were consecutively included in a prospective registry. Trans-oesophageal echocardiography (TEE) and clinical neurological assessment were performed 3-9 months after the procedure and follow-up was updated henceforth by telephone. Patients were divided into 2 groups according to the device: NDAs or ACP.

\section{Procedure}

TEE was performed prior to LAA closure for exclusion of LAA thrombi. LAA closure was performed under local anaesthesia and fluoroscopic guidance only. Unless a PFO or ASD were present for passage, trans-septal needle puncture was performed under fluoroscopic guidance in anteroposterior and lateral views (fig. 4). Multi-plane X-ray graphy of the LAA was performed to assess the diameter of its neck (landing zone), to select the device size and - in the NDA group - the device type. The implant was then deployed into the LAA by retraction of the delivery sheath after having advanced the leading anchoring part of the device (left disc or lobe) out of it. After full deployment, sufficient anchoring was assured by a well dosed and sustaining tug on the delivery cable. The disc in the left atrium had to move freely, while the anchoring disc (NDA) or lobe (ACP) moved only in unison with the LAA. If the implant location and anchoring were not satisfactory, the device was recaptured and redeployed or a different size or shape (for NDA) was selected. An injection of contrast medium in several projections showing 2 discs or the lobe and the disc, respectively, in profile without overlap was performed before device release to assess the correct position. After releasing the device, the sheath was removed and haemostasis was achieved by manual compression performed by the patient unless an arterial puncture had also been performed [21].

The omission of intra-procedural TEE for measurement or guidance offers the advantage of a more frugal, short, and patient-friendly procedure without need for general anaesthesia or deep sedation. Time is important to avoid clots in the gear, particularly when combining procedures (additional coronary or other structural interventions). Trans-thoracic echocardiography was performed before hospital discharge within 24 hours of trans-catheter LAA occlusion to exclude device embolisation, pericardial effusion, or other serious complications. OAC was not continued after implantation. Patients were maintained on ASA $100 \mathrm{mg}$ daily for 3-6 months and clopidogrel $75 \mathrm{mg}$ daily for 1 month, unless long-term anti-platelet therapy was required for another reason.

\section{Devices}

The NDAs feature two flat double discs (40-56 mm) of a nitinol mesh and polyester fabric with necks of variable diameters and lengths. Risk for embolisation represents the main bane of NDAs. The ACP is a self-expanding device, representing a modification of the Amplatzer double-disc septal occluders and is delivered through a double-curved trans-septal access sheath. The ACP is composed of a lobe and a disc connected by a flexible central waist. The lobe is equipped with tiny hooks to enhance anchorage in the LAA. The disc (a remnant of the NDAs) is meant to seal the orifice of the LAA according to the pacifier principle (fig. 1-2). The ACP is available in eight lobe diameters $(16-30 \mathrm{~mm})$, and the disc exceeds that by $4-6 \mathrm{~mm}$.

\section{Statistical analysis}

Continuous variables are expressed as mean \pm standard deviation. Comparisons between both groups were performed by a 2 -sided, unpaired t test. Categorical variables are reported as counts and percentages and compared by Chi square test. Due to small sample sizes with non-normal distribution, a non-parametric Mann-Whitney U-test was performed. Statistical significance was assumed with a $p$ value $<0.05$. All data were analysed by SPSS software version 15 (SPSS Inc., Chicago, Illinois). 


\section{Figure 4}

Steps of ACP implantation: (1.) Septal puncture, simultaneous contrast medium injection depicts localisation and thickness of the inter-atrial septum. (2.) Opacification of the left atrial appendage (LAA), (3.) Deployment of lobe (anchoring plug). (4.) Deployment of disc. (5.) Proof of sufficient anchorage by tug test. Lobe stays put. (6.) Proof of LAA orifice sealing according to the pacifier principle. (7.) Disconnection from the delivery cable. (8.) Trans-septal LAA and patent foramen ovale (PFO) closure in one session. (9.) Pre-existing septal occluder guides later trans-septal puncture. $\mathrm{LA}=$ left atrium; RA = right atrium; LAA = left atrial appendage; PFO = patent foramen ovale.

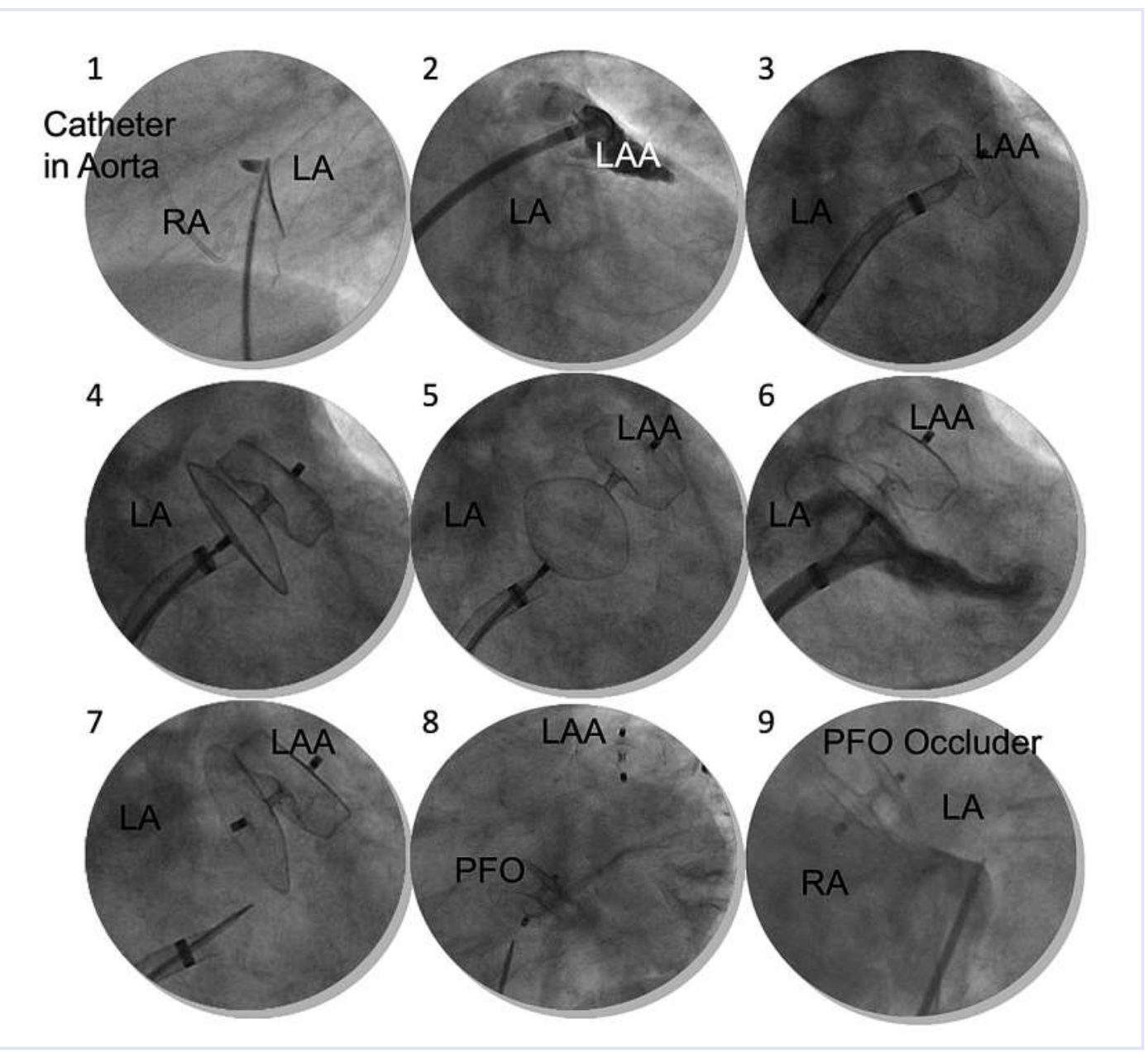

\section{Results}

The baseline variables are depicted in table 1. In the first 32 patients receiving an Amplatzer NDA, a prototype of the currently available ACP without retention hooks was implanted in one patient. In the subsequent 32 patients, an ACP was implanted. Age and gender distribution were similar in both groups.

$\mathrm{CHADS}_{2}$ and $\mathrm{CHA}_{2}$ VASc scores were lower in the NDA group, than in the ACP group. Prior to LAA closure, $72 \%$ of patients in the NDA group were on OAC compared to $50 \%$ in the ACP group despite higher $\mathrm{CHADS}_{2}$ scores in the ACP group $(p=0.07)$.

The use of blood thinners is shown in figure 5 . During follow-up, 1 patient in the NDA group resumed OAC compared to 2 patients in the ACP group. The indication for restarting OAC after LAA occlusion was a thrombus on the device in 2 patients and atrial flutter ablation in 1 patient.
Tables 2 and 3 depict procedural results, complications, and follow-up data. Of the five device embolisations (four with ASD, one with a PFO device, all NDA group and all in atrial fibrillation at time of intervention), four occurred intra-procedurally and one after leaving the laboratory (diagnosed at six months). In two cases, surgical removal of devices (one from the left atrium, one from the left ventricle) was performed along with surgical closure of the LAA. In 3 cases, the devices were removed from the descending aorta, 2 by vascular surgery in the groin and one by percutaneous removal. In the ACP group, no embolisations occurred. Two patients in the NDA group developed cardiac tamponade resolved by pericardiocentesis. In one patient, an insignificant pericardial effusion was diagnosed on the post-procedural day but required no action.

Mean mid-term follow-up was performed $7.2 \pm$ 2.7 months after LAA closure. In 15 patients in the NDA group, follow-up TEE was not performed compared to 
Table 1

Baseline variables.

\begin{tabular}{|c|c|c|c|c|c|c|c|}
\hline & \multicolumn{2}{|l|}{ Total } & \multicolumn{2}{|c|}{ NDA $n=32$} & \multicolumn{2}{|c|}{$A C P n=32$} & \multirow[t]{2}{*}{ p } \\
\hline & n & $\%$ & $\mathbf{n}$ & $\%$ & $\mathbf{n}$ & $\%$ & \\
\hline Male & 44 & 69 & 23 & 72 & 21 & 66 & 0.22 \\
\hline Age at implantation & $66 \pm 9$ & & $63 \pm 7$ & & $69 \pm 10$ & & 0.05 \\
\hline Height (cm) & $172 \pm 7$ & & $175 \pm 8$ & & $170 \pm 9$ & & 0.02 \\
\hline Weight (kg) & $83 \pm 16$ & & $83 \pm 17$ & & $83 \pm 15$ & & 0.93 \\
\hline \multicolumn{8}{|l|}{ Atrial rhythm } \\
\hline Paroxysmal fibrillation & 37 & 58 & 13 & 41 & 24 & 75 & 0.01 \\
\hline Persistent/permanent fibrillation & 26 & 41 & 19 & 59 & 7 & 22 & $<0.01$ \\
\hline Flutter & 1 & 2 & 0 & 0 & 1 & 3 & 0.31 \\
\hline \multicolumn{8}{|l|}{ CHADS $_{2}$ Score } \\
\hline Congestive heart failure & 7 & 11 & 1 & 3 & 6 & 19 & 0.05 \\
\hline Hypertension & 39 & 61 & 15 & 47 & 24 & 75 & 0.02 \\
\hline Age & 16 & 25 & 1 & 3 & 15 & 47 & $<0.01$ \\
\hline Diabetes & 10 & 16 & 1 & 3 & 9 & 28 & 0.01 \\
\hline Stroke & 20 & 31 & 7 & 22 & 13 & 41 & 0.11 \\
\hline Total & $1.75 \pm 1.4$ & & $1.0 \pm 1.0$ & & $2.5 \pm 1.3$ & & 0.44 \\
\hline \multicolumn{8}{|l|}{ Main Indication for LAA closure } \\
\hline Major bleeding or high risk of bleeding & 34 & 53 & 12 & 38 & 22 & 69 & 0.01 \\
\hline History of falls & 4 & 6 & 1 & 3 & 3 & 9 & 0.30 \\
\hline INR 2-3 not achievable & 6 & 9 & 3 & 9 & 3 & 9 & \\
\hline Physician or patient refusal of Vitamin $\mathrm{K}$ antagonist & 20 & 31 & 16 & 50 & 4 & 13 & $<0.01$ \\
\hline \multicolumn{8}{|l|}{ Medication before LAA closure } \\
\hline Acetylsalicylic acid & 20 & 31 & 5 & 16 & 15 & 47 & 0.01 \\
\hline Thienopyridine & 4 & 6 & 0 & 0 & 4 & 13 & 0.04 \\
\hline Dual anti-platelet therapy & 3 & 5 & 0 & 0 & 3 & 9 & 0.08 \\
\hline Vitamin $\mathrm{K}$ antagonists & 39 & 61 & 23 & 72 & 16 & 50 & 0.07 \\
\hline
\end{tabular}

\section{Figure 5}

Medication before and 3-6 months after left atrial appendage occlusion. ASA = acetylsalicylic acid; $A C P=$ atrial cardiac plug;

NDA $=$ non-dedicated Amplatzer occluders

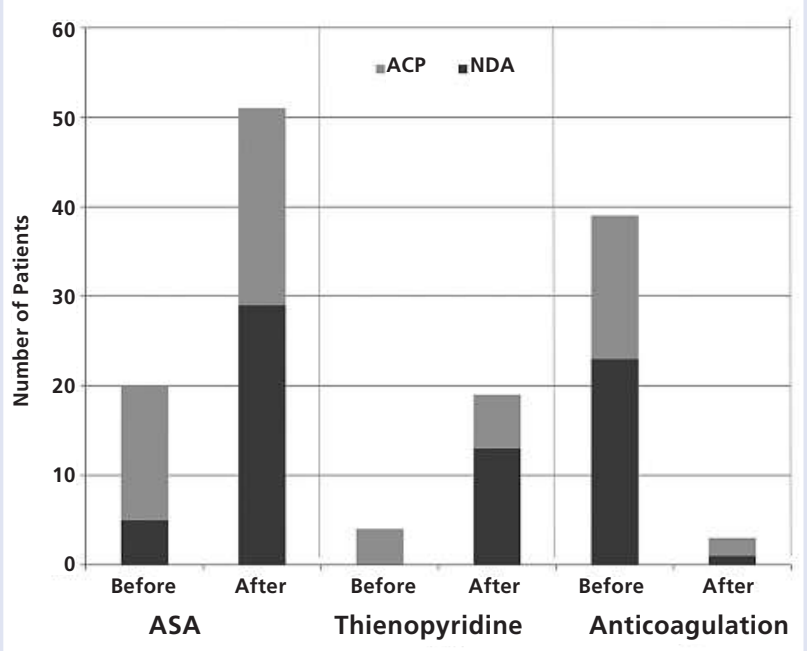

four patients in the ACP group. Reasons were co-morbidities, embolisation of the device (5 NDA, $0 \mathrm{ACP}$ ) and patient or referring physician refusal. TEE showed incomplete LAA closure (color Doppler jet width $>5 \mathrm{~mm}$ ) in 3 out of 17 patients (18\%) in the NDA group, compared to 1 out of 28 patients (4\%) in the ACP group ( $p=0.11$ ) without further treatment. In 1 of 17 patients in the NDA group (6\%), a mobile thrombus was shown. This patient refused re-initiation of OAC. Two and a half years later clot embolisation to a leg needed surgical treatment. In 1 of 17 patients $(6 \%)$ in the NDA group and 2 of 28 (7\%) in the ACP group, a non-mobile thrombus was shown and OAC was restarted for 3-4 months, after which the thrombus had shrunk or disappeared on repeat TEE. In 4 of 28 patients of the ACP group (14\%), a potential non-mobile thrombus attached to the disc could not be ruled out. Two patients were already on ASA and no further anti-thrombotics were deemed indicated. In one patient ASA was started 
Table 2

Procedural variables.

\begin{tabular}{|c|c|c|c|c|c|c|c|}
\hline & Total & & NDA & & $\mathrm{ACP}$ & & $p$ \\
\hline & $\mathbf{n}$ & $\%$ & $\mathbf{n}$ & $\%$ & $\mathbf{n}$ & $\%$ & \\
\hline \multicolumn{8}{|l|}{ Intervention } \\
\hline \multicolumn{8}{|l|}{ Occluder } \\
\hline ASD & 23 & 36 & 23 & 72 & 0 & 0 & \\
\hline VSD & 6 & 9 & 6 & 19 & 0 & 0 & \\
\hline PFO & 2 & 3 & 2 & 6 & 0 & 0 & \\
\hline Prototype & 1 & 2 & 1 & 3 & 0 & 0 & \\
\hline Amplatzer Cardiac Plug & 32 & 50 & 0 & 0 & 32 & 100 & \\
\hline Fluoroscopy time (min) & \multicolumn{2}{|l|}{$23 \pm 18$} & \multicolumn{2}{|l|}{$28 \pm 13$} & \multicolumn{2}{|l|}{$19 \pm 20$} & 0.06 \\
\hline Contrast medium (ml) & \multicolumn{2}{|l|}{$325 \pm 189$} & \multicolumn{2}{|l|}{$424 \pm 182$} & \multicolumn{2}{|l|}{$226 \pm 134$} & $<0.01$ \\
\hline More than 1 device tried & 5 & 8 & 3 & 9 & 2 & 6 & 0.64 \\
\hline \multicolumn{8}{|l|}{ Incidental intervention } \\
\hline Coronary angiography & 47 & 74 & 29 & 91 & 18 & 56 & $<0.01$ \\
\hline $\mathrm{PCl}$ & 7 & 11 & 1 & 3 & 6 & 19 & 0.05 \\
\hline ASD/PFO closure & 16 & 25 & 5 & 16 & 11 & 34 & 0.08 \\
\hline Ablation of atrial fibrillation & 9 & 14 & 3 & 9 & 6 & 19 & 0.28 \\
\hline Hospital stay (days) & \multicolumn{2}{|l|}{$1.6 \pm 1.9$} & \multicolumn{2}{|l|}{$1.3 \pm 0.5$} & \multicolumn{2}{|l|}{$1.9 \pm 2.5$} & $<0.01$ \\
\hline Same-day procedure & 5 & 8 & 0 & 0 & 5 & 16 & 0.02 \\
\hline
\end{tabular}

and continued indefinitely, in one patient OAC was initiated and kept for 4 months, after which, however, no change was seen in the repeat TEE.

Neurological clinical follow-up did not reveal new deficits in any patient. A cerebrovascular accident did not occur during the follow-up period for any of the 64 patients. No major bleedings were observed in any patient.

Long-term follow-up was complete in $100 \%$ of the NDA group (mean $75 \pm 16$ months, 199 patient-years). A total of 5 patients died during follow-up (2 cancer, 1 heart failure, 1 pulmonary embolism, and 1 of unknown reason one year after LAA closure). The ACP patients yielded 9 patient-years of follow-up, with no deaths.

\section{Discussion}

The study demonstrates that trans-catheter LAA closure with Amplatzer devices is fairly safe and efficacious with acceptable peri-interventional complication rates and good mid and long-term outcome. There was no device-related mortality. The new ACP closure device showed superior success and safety.

Abstracting from technical problems (mainly device embolisation), the off-label use of NDAs shows good clinical results. Based on the $\mathrm{CHADS}_{2}$ score [23], they are at least competitive to OAC (fig. 6). In our study, only one event happened in the NDA group as described above.

\section{Figure 6}

With the CHADS 2 score of 1.0 in the NDA group, 5.5 cerebrovascular accidents (CVA) (100\%) would have been expected during follow-up of 199 patient-years (long-term follow-up). With oral anticoagulation (OAC), a $64 \%$ reduction is anticipated (1.8 events; $36 \%)$. After LAA occlusion only 1 event happened (18\%).

$\mathrm{ACP}=$ atrial cardiac plug;

NDA $=$ non-dedicated Amplatzer occluders

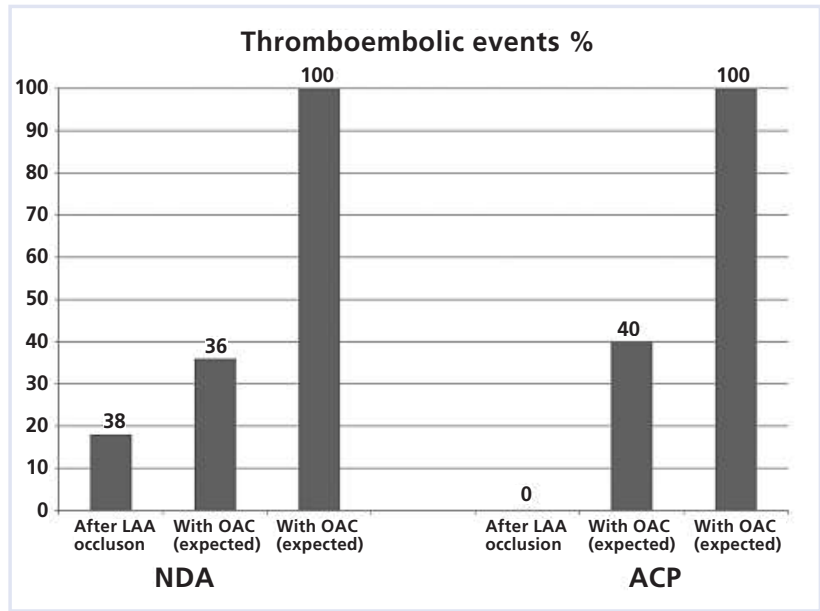


Table 3

Procedural results and follow-up.

\begin{tabular}{|c|c|c|c|c|c|c|c|}
\hline & \multicolumn{2}{|l|}{ Total } & \multicolumn{2}{|l|}{ NDA } & \multicolumn{2}{|l|}{ ACP } & \multirow[t]{2}{*}{$\mathbf{p}$} \\
\hline & $\mathbf{n}$ & $\%$ & $\mathbf{n}$ & $\%$ & $\mathrm{n}$ & $\%$ & \\
\hline Procedural success & 59 & 92 & 27 & 84 & 32 & 100 & 0.02 \\
\hline Device embolisation & 5 & 8 & 5 & 16 & 0 & 0 & 0.02 \\
\hline Femoral arteriovenous fistula & 2 & 3 & 2 & 6 & 0 & 0 & 0.15 \\
\hline Pericardial effusion & 3 & 5 & 2 & 6 & 1 & 3 & 0.55 \\
\hline Requiring pericardiocentesis & 2 & 3 & 2 & 6 & 0 & 0 & 0.15 \\
\hline Requiring surgery & 0 & 0 & 0 & 0 & 0 & 0 & \\
\hline Air embolism & 2 & 3 & 1 & 3 & 1 & 3 & \\
\hline Clot embolism & 1 & 2 & 1 & 3 & 0 & 0 & 0.31 \\
\hline \multicolumn{8}{|l|}{ Mid-term follow-up } \\
\hline Stroke & $0 / 64$ & 0 & $0 / 32$ & 0 & $0 / 32$ & 0 & \\
\hline Incomplete closure of LAA on TEE & $4 / 45$ & 9 & $3 / 17$ & 18 & $1 / 28$ & 4 & 0.11 \\
\hline Pericardial effusion on TEE & 0 & 0 & 0 & 0 & 0 & 0 & \\
\hline \multicolumn{8}{|l|}{ Thrombus on device on TEE } \\
\hline Mobile & $1 / 45$ & 2 & $1 / 17$ & 6 & $0 / 28$ & 0 & 0.19 \\
\hline Non-mobile & $3 / 45$ & 7 & $1 / 17$ & 6 & $2 / 28$ & 7 & 0.87 \\
\hline Possible & $4 / 45$ & 9 & $0 / 17$ & 0 & $4 / 28$ & 14 & 0.10 \\
\hline Death (device related) & 0 & 0 & 0 & 0 & 0 & 0 & 0 \\
\hline \multicolumn{8}{|l|}{ Medication at mid-term follow up } \\
\hline Acetylsalicylic acid & $51 / 64$ & 80 & $29 / 32$ & 91 & $22 / 32$ & 68 & 0.03 \\
\hline Thienopyiridine & $19 / 64$ & 30 & $13 / 32$ & 41 & $6 / 32$ & 19 & 0.06 \\
\hline Coumadin resumed later & $3 / 64$ & 5 & $1 / 32$ & 3 & $2 / 32$ & 6 & 0.55 \\
\hline
\end{tabular}

Both groups differed significantly with respect to baseline characteristics. Despite higher rates of cardiovascular co-morbidities of the patients in the ACP group, clinical outcome was similar.

The remarkably high rate of physician or patient refusal of OAC as an indication for LAA closure occurred spontaneously. These patients had no protection other than platelet inhibitors in some. Against that background, LAA occlusion with an off-label device was deemed an ethical and promising therapy.

The ACP with its hooks and its double-curved sheath rendered the procedure more operator-friendly, increased technical success, and reduced complications. In spite of that, a recently published European multicenter ACP registry [21] including 143 patients demonstrated higher failure rates and complications compared to our results. This may point out the fact that a learning curve for the technique of transcatheter LAA exclusion as a whole is influential. The significant reduction of contrast medium and shortening of fluoroscopy time reflects these improvements, particularly in light of a higher rate of additional interventions in the ACP group.
The slow blood flow in the fibrillating and commonly enlarged left atrium is thrombogenic. It comes as no surprise that device-associated thrombi were detected in both groups. The higher rate of thrombi suspected in the ACP group may be explained by the particularly thrombogenic milieu proven by the higher CHADS and $\mathrm{CHA}_{2} \mathrm{DS}_{2}$-VASc scores of this group. Yet, these thrombi were mainly firmly attached to the disc (sort of filling in gaps when the disc was partially within the LAA rather than outside its orifice) and there were no clinical manifestations.

New anticoagulants may be easier to administer, more effective, and less prone to bleeding but they still carry a risk of major haemorrhage. To answer the critical question of whether LAA occlusion with the ACP may be non-inferior or even superior to chronic anticoagulation with warfarin or its successors dabigatran, rivaroxaban, or apixaban, prospective, randomised trials will be needed. Their results will be of pivotal impact for the entire concept of LAA occlusion, since device implantation harbours risks of serious complications. Finally a co-existence of LAA closure and OAC is possible as shown in some of our cases. It offers a third option in selected patients. 
For the short-term, radiofrequency ablation of AF foci offers a $77 \%$ success rate. In the long-term, however, the rate of recurrence remains high [24], anticoagulation has generally to be continued and long-term data regarding rates of systemic embolism are lacking.

\section{Study limitations}

The study consisted of small sample sizes and was not randomised. In addition, the patients were not matched. Thus we cannot exclude several forms of bias such as operator experience and group in-homogeneity. Since no long-term results are available yet for the ACP, the current results can only be extrapolated on the basis of the mid-term results and the long-term results of the NDA group.

\section{Conclusion}

Trans-catheter LAA closure with ACP is safer and technically easier with lower peri-interventional complication rates than with NDAs. Good long-term results can be expected with ACPs based on experience obtained with NDAs. Further studies are needed to demonstrate trans-catheter LAA closure to be at least as good as OAC for stroke prevention in patients with $\mathrm{AF}$, with or without contraindications for OAC. Finally, the combination of OAC and device closure has to be looked at as neither of the two is perfect.

\section{References}

1 Heeringa J, van der Kuip DA, Hofman A, Kors JA, van Herpen G, Stricker BH, et al. Prevalence, incidence and lifetime risk of atrial fibrillation: the Rotterdam study. Eur Heart J. 2006;27:949-53.

2 Schnabel RB, Sullivan LM, Levy D, Pencina MJ, Massaro JM, D'Agostino RB, Sr., et al. Development of a risk score for atrial fibrillation (Framingham Heart Study): a community-based cohort study. Lancet. 2009;373:739-45.

3 Go AS, Hylek EM, Phillips KA, Chang Y, Henault LE, Selby JV, Singer DE. Prevalence of diagnosed atrial fibrillation in adults: national implications for rhythm management and stroke prevention: the AnTicoagulation and Risk Factors in Atrial Fibrillation (ATRIA) Study. JAMA. 2001;285:2370-5.

4 Gage BF, Waterman AD, Shannon W, Boechler M, Rich MW, Radford MJ. Validation of clinical classification schemes for predicting stroke: results from the National Registry of Atrial Fibrillation. JAMA. 2001; 285:2864-70.

5 Weera Gregory Y. H. Lip, MD; Robby Nieuwlaat, PhD; Ron Pisters, MD; Deirdre A. Lane, PhD; and Harry J. G. M. Crijns, MD. Refining Clinical Risk Stratification for Predicting Stroke and Thromboembolism in Atrial Fibrillation Using a Novel Risk Factor-Based Approach. The Euro Heart Survey on Atrial Fibrillation. Chest. 2010;137(2):263-72.

6 Aberg H. Atrial fibrillation. I. A study of atrial thrombosis and systemic embolism in a necropsy material. Acta Med Scand. 1969;185:373-9.

7 Blackshear JL, Odell JA. Appendage obliteration to reduce stroke in cardiac surgical patients with atrial fibrillation. Ann Thorac Surg. 1996; 61:755-9.
8 Manning WJ, Weintraub RM, Waksmonski CA, Haering JM, Rooney PS, Maslow AD, et al. Accuracy of transesophageal echocardiography for identifying left atrial thrombi. A prospective, intraoperative study. Ann Intern Med. 1995;123:817-22.

9 Tsai LM, Chen JH, Lin LJ, Yang YJ. Role of transesophageal echocardiography in detecting left atrial thrombus and spontaneous echo contrast in patients with mitral valve disease or non-rheumatic atrial fibrillation. J Formos Med Assoc. 1990;89:270-4.

10 Mant J, Hobbs FD, Fletcher K, Roalfe A, Fitzmaurice D, Lip GY, Murray $\mathrm{E}$. Warfarin versus aspirin for stroke prevention in an elderly community population with atrial fibrillation (the Birmingham Atrial Fibrillation Treatment of the Aged Study, BAFTA): a randomised controlled trial. Lancet. 2007;370:493-503.

11 van Walraven C, Hart RG, Connolly S, Austin PC, Mant J, Hobbs FD, et al. Effect of age on stroke prevention therapy in patients with atrial fibrillation: the atrial fibrillation investigators. Stroke. 2009; 40:1410-6.

12 Rockson SG, Albers GW. Comparing the guidelines: anticoagulation therapy to optimize stroke prevention in patients with atrial fibrillation. J Am Coll Cardiol. 2004;43:929-35.

13 Sacco RL, Adams R, Albers G, Alberts MJ, Benavente O, Furie K, et al. Guidelines for prevention of stroke in patients with ischemic stroke or transient ischemic attack: a statement for healthcare professionals from the American Heart Association/American Stroke Association Council on Stroke: co-sponsored by the Council on Cardiovascular Radiology and Intervention: the American Academy of Neurology affirms the value of this guideline. Stroke. 2006;37:577-617.

14 Landefeld CS, Beyth RJ. Anticoagulant-related bleeding: clinical epidemiology, prediction, and prevention. Am J Med. 1993;95:315-28.

15 Bungard TJ, Ghali WA, Teo KK, McAlister FA, Tsuyuki RT. Why do patients with atrial fibrillation not receive warfarin? Arch Intern Med. 2000;160:41-6.

16 Healey JS, Hart RG, Pogue J, Pfeffer MA, Hohnloser SH, De Caterina R, et al. Risks and benefits of oral anticoagulation compared with clopidogrel plus aspirin in patients with atrial fibrillation according to stroke risk: the atrial fibrillation clopidogrel trial with irbesartan for prevention of vascular events (ACTIVE-W). Stroke. 2008;39:1482-6.

17 Johnson WD, Ganjoo AK, Stone CD, Srivyas RC, Howard M. The left atrial appendage: our most lethal human attachment! Surgical implications. Eur J Cardiothorac Surg. 2000;17:718-22.

18 Garcia-Fernandez MA, Perez-David E, Quiles J, Peralta J, GarciaRojas I, Bermejo J, et al. Role of left atrial appendage obliteration in stroke reduction in patients with mitral valve prosthesis: a transesophageal echocardiographic study. J Am Coll Cardiol. 2003;42:1253-8.

19 Sievert H, Lesh MD, Trepels T, Omran H, Bartorelli A, Della Bella P, et al. Percutaneous left atrial appendage transcatheter occlusion to prevent stroke in high-risk patients with atrial fibrillation: early clinical experience. Circulation. 2002;105:1887-9.

20 Meier B, Palacios I, Windecker S, Rotter M, Cao QL, Keane D, et al. Transcatheter left atrial appendage occlusion with Amplatzer devices to obviate anticoagulation in patients with atrial fibrillation. Catheter Cardiovasc Interv. 2003;60:417-22.

21 Park JW, Bethencourt A, Sievert H, Santoro G, Meier B, Walsh K, et al. Left atrial appendage closure with amplatzer cardiac plug in atrial fibrillation - Initial European experience. Catheter Cardiovasc Interv. 2011;77:700-6.

22 Holmes DR, Reddy VY, Turi ZG, Doshi SK, Sievert H, Buchbinder M, et al. Percutaneous closure of the left atrial appendage versus warfarin therapy for prevention of stroke in patients with atrial fibrillation: a randomised non-inferiority trial. Lancet. 2009;374:534-42.

23 Hart RG, Pearce LA, Aguilar MI. Meta-analysis: antithrombotic therapy to prevent stroke in patients who have nonvalvular atrial fibrillation. Ann Intern Med. 2007;146:857-67.

24 Weerasooriya R, Khairy P, Litalien J, Macle L, Hocini M, Sacher F, et al. Catheter ablation for atrial fibrillation: are results maintained at 5 years of follow-up? J Am Coll Cardiol. 57:160-6. 